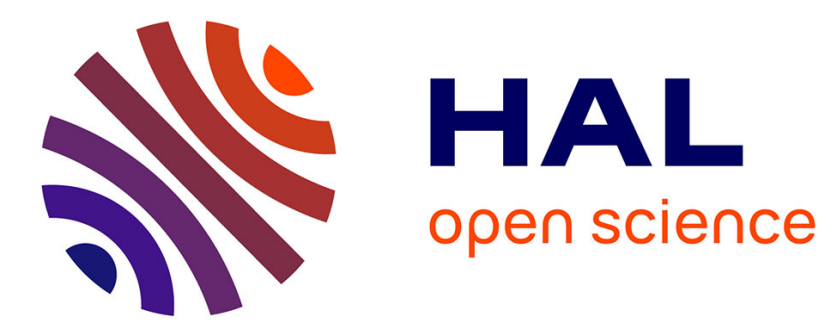

\title{
Robust observer design for uncertain Takagi-Sugeno model with unmeasurable decision variables: an L2 approach
}

\author{
Dalil Ichalal, Benoît Marx, José Ragot, Didier Maquin
}

\section{To cite this version:}

Dalil Ichalal, Benoît Marx, José Ragot, Didier Maquin. Robust observer design for uncertain Takagi-Sugeno model with unmeasurable decision variables: an L2 approach. 16th Mediterranean Conference on Control and Automation, MED'08, Jun 2008, Ajaccio, France. pp.274-279, 10.1109/MED.2008.4602078 . hal-00292256

\section{HAL Id: hal-00292256 \\ https://hal.science/hal-00292256}

Submitted on 5 May 2014

HAL is a multi-disciplinary open access archive for the deposit and dissemination of scientific research documents, whether they are published or not. The documents may come from teaching and research institutions in France or abroad, or from public or private research centers.
L'archive ouverte pluridisciplinaire HAL, est destinée au dépôt et à la diffusion de documents scientifiques de niveau recherche, publiés ou non, émanant des établissements d'enseignement et de recherche français ou étrangers, des laboratoires publics ou privés. 


\title{
Robust observer design for uncertain Takagi-Sugeno model with unmeasurable decision variables: an $\mathscr{L}_{2}$ approach
}

\author{
Dalil Ichalal, Benoît Marx, José Ragot and Didier Maquin
}

\begin{abstract}
This paper deals with the problem of state estimation of nonlinear uncertain systems described by uncertain multiple model form with unmeasurable decision variables. We propose two methods to attenuate the effect of modeling uncertainties and measurement noise on the state estimation. The first method is based, under some assumptions, on the second method of Lyapunov and $\mathscr{L}_{2}$ approach. The second method allows to reduce the conservatism of the convergence conditions issued from the assumptions of first method. The convergence conditions of the observer are presented in terms of linear matrix inequality (LMI) formulation. The validity and applicability of the proposed methods are illustrated by an academic example.
\end{abstract}

\section{INTRODUCTION}

The design of observers for the state estimation is an important problem in the automatic control domain with diverse areas of application. Indeed, several issues require system's state reconstruction, such as designing a state feedback control law or constructing a diagnosis system in order to monitor and reconfigure the system in the case of occurrence of failures. Observers are also used to estimate the states which are not accessible, or to replace sensors that are bulky, expensive and difficult to maintain.

Among the solutions to the problem of the state estimation is the famous observer proposed by Luenberger in 1971 [1], for time-invariant linear systems. This observer is based on the synthesis of a static gain in order to stabilize the state estimation error and ensure its asymptotic convergence. However, the presence of disturbances or noise causes a bad reconstruction of the system's state. Another method, for linear systems, which takes into account the measurement noise affecting output and the state of the system, was proposed by Kalman in 1960 [2]. Based on the statistical knowledge of noises, this method allows to reconstruct the state of the system in the presence of measurement noise, and it calculates at each time, the gain of the filter. In order to improve the performance of observers, a lot of works have been carried out to develop observers which are robust against uncertainties of modeling and external disturbances such as noises or faults.

One of the best methods used successfully in practice is the unknown input observer (UIO), based on decoupling the unknown input and the state estimation error, which allows to

This research was supported by the TASSILI No. 07 program under MDU grant 714 .

The authors are with Centre de Recherche en Automatique de Nancy (CRAN), Nancy-Université, CNRS, 2 avenue de la Forêt de Haye F-54516 Vandoeuvre-les-Nancy. \{dalil.ichalal, benoit.marx, jose.ragot, didier.maquin\}eensem.inpl-nancy.fr eliminate the effect of this disturbance on the reconstruction error. Among this kind of methods, the important work published in [3] in 1975, where the authors propose a minimal order UIO for a linear system in the presence of known and unknown inputs can be mentioned. This work was followed by several works on the problems of the state estimation with unknown input. When the conditions of application of the previously mentioned methods are not satisfied, alternative methods can be used [4], [5]. They are based on the use of $H_{\infty}$ techniques to attenuate the effect of measurement noise and disturbances on the state estimation.

In the context of diagnosis of linear systems, the observers are used as residual generators. In [6] [7], the authors investigate this problem by using robust state estimation methods to generate residual signals which are sensitive to faults and insensitive to disturbances and noises. The detection and isolation of sensor and actuator faults are often carried out by the construction of banks of observers.

Unfortunately, all of the works cited above relate to the class of linear systems only. However, this assumption of linearity limits the domain of application of these methods and reduces their performances. So, in recent years, several studies have been conducted on specific classes of nonlinear systems. However, the study of nonlinear systems described by the general form $\dot{x}=f(x, u, t)$ is often difficult because of the complexity of the function $f$. The most studied class in the literature is the class of Lipschitz systems, represented by a linear part and a nonlinear part which satisfies the Lipschitz property [8] [9] [10].

The works carried out on fuzzy systems in [11] allow to get a more interesting representation of nonlinear dynamical systems. This representation is based on the interpolation of linear local models, representing the local behaviors of the nonlinear system, by nonlinear weighting functions. The modeling ability of the Takagi-Sugeno model (also called multiple model) lies in its property of universal approximator. Its simple representation inspired with linear systems allows to generalize synthesis and analysis tools developed for linear systems to the case of nonlinear systems. For example, [12] proposed a study of stability and stabilization by multiple controllers. One can also find in [13], tools directly inspired by the study of linear systems in order to study the stability and stabilization of nonlinear systems described by multiple model form. In [14], Patton proposed an observer based on the Luenberger observer structure, and applied for diagnosis. In [15], the sliding mode observer developed for linear systems has been extended for nonlinear systems described by a multiple model in the presence of unknown input and 
modeling uncertainties.

\section{PRELIMINARIES AND PROBLEM STATEMENT}

\section{A. Multiple model approach}

Consider the following general form of continuous-time nonlinear systems:

$$
\left\{\begin{array}{l}
\dot{x}(t)=f(x(t), u(t)) \\
y(t)=h(x(t), u(t))
\end{array}\right.
$$

where $x \in \mathbb{R}^{n}, u \in \mathbb{R}^{m}, y \in \mathbb{R}^{q}$ and $f$ and $h$ are nonlinear functions. The representation (1) is very difficult to study, elsewhere in literature, all of the works developed on the nonlinear systems concern specific classes for example Lipschitz systems which are represented by a linear part and a nonlinear one. The nonlinear part is assumed to be Lipschitz with respect to $x$.

As mentioned in section 1, the multiple model approach is a very interesting method to represent nonlinear systems. The general form of a multiple model structure is given by:

$$
\left\{\begin{aligned}
\dot{x}(t) & =\sum_{i=1}^{r} \mu_{i}(\xi(t))\left(A_{i} x(t)+B_{i} u(t)\right) \\
y(t) & =\sum_{i=1}^{r} \mu_{i}(\xi(t))\left(C_{i} x(t)+D_{i} u(t)\right)
\end{aligned}\right.
$$

where $A_{i} \in \mathbb{R}^{n \times n}, B_{i} \in \mathbb{R}^{n \times m}, C_{i} \in \mathbb{R}^{q \times n}$ and $D_{i} \in \mathbb{R}^{q \times m}$. The weighing functions $\mu_{i}$ are nonlinear and depend on the decision variable $\xi(t)$ which can be measurable like $\{u(t), y(t)\}$ or unmeasurable like the state $x(t)$ of the system. The weighting functions satisfy the following properties:

$$
\left\{\begin{array}{c}
0 \leq \mu_{i}(\xi(t)) \leq 1 \\
\sum_{i=1}^{r} \mu_{i}(\xi(t))=1
\end{array}\right.
$$

This structure is simple and represents a universal approximator since it can represent any nonlinear behavior. The multiple model structure provides a mean to generalize the tools developed for linear systems to nonlinear systems.

\section{B. Problem statement}

In almost all of the works cited in the introduction, the authors assume that the weighting functions depend on measurable decision variables i.e $\xi(t)=u(t)$ or $\xi(t)=y(t)$. In the context of diagnosis of nonlinear systems, this hypothesis requires designing observers with weighting functions which depend on the input $u(t)$, for the detection of sensor faults, or which depend on the output $y(t)$ in order to detect actuator faults. Indeed, if one uses $\xi(t)=u(t)$ for example, in the observer banks, the $i^{t h}$ observer does not use the $u_{i}$ input. However, the knowledge of $u_{i}$ is required in the evaluation of the weighting functions. Consequently the state or output estimation is not possible and it becomes impossible to locate a fault. For this reason, it is interesting to consider the case where the weighting functions depend on unmeasurable variables such as the state of the system, and thus, the weighting functions in the observer will depend on the estimated state. In this context, there are few works, nevertheless, we can cite [16], [17] and [18], where the authors proposed an observer, using the assumption of Lipschitz property of the considered perturbed term. The conditions of convergence of the observer are expressed in terms of Linear Matrix Inequalities (LMI) [19]. Unfortunately, the Lipschitz constants of the weighting functions appear in the LMI and reduce the domain of applicability of the method. Indeed, if these constants get large values, it is possible that the LMIs cannot have solutions.

In this paper, we propose two methods to design observers for nonlinear systems described by an uncertain multiple model form (Takagi-Sugeno) with unmeasurable decision variables $(\xi(t)=x(t))$.

\section{MAIN RESULTS}

Let us consider the following uncertain multiple model with unmeasurable decision variables:

$$
\left\{\begin{array}{l}
\dot{x}=\sum_{i=1}^{r} \mu_{i}(x)\left(\left(A_{i}+\Delta A_{i}\right) x+\left(B_{i}+\Delta B_{i}\right) u\right) \\
y=C x+D \omega
\end{array}\right.
$$

where:

$$
\begin{aligned}
\Delta A_{i}(t) & =M_{i}^{A} \Sigma_{A}(t) N_{i}^{A} \\
\Delta B_{i}(t) & =M_{i}^{B} \Sigma_{B}(t) N_{i}^{B}
\end{aligned}
$$

with:

$$
\begin{aligned}
& \Sigma_{A}^{T}(t) \Sigma_{A}(t) \leq I, \quad \forall t \\
& \Sigma_{B}^{T}(t) \Sigma_{B}(t) \leq I, \quad \forall t
\end{aligned}
$$

where $I$ is the identity matrix and $\omega(t)$ is a bounded measurement noise.

\section{A. First approach}

By introducing the matrix $A_{0}$ defined as follows:

$$
A_{0}=\frac{1}{r} \sum_{i=1}^{r} A_{i}
$$

and:

$$
A_{i}=\bar{A}_{i}+A_{0}
$$

we obtain another formulation of the system (4):

$$
\left\{\begin{array}{l}
\dot{x}=A_{0} x+\sum_{i=1}^{r} \mu_{i}(x)\left(\left(\bar{A}_{i}+\Delta A_{i}\right) x+\left(B_{i}+\Delta B_{i}\right) u\right) \\
y=C x+D \omega
\end{array}\right.
$$

We are interested in designing an observer in order to estimate the state of the system presented above. The observer is described by the following form:

$$
\left\{\begin{array}{l}
\dot{\hat{x}}=A_{0} \hat{x}+\sum_{i=1}^{r} \mu_{i}(\hat{x})\left(\bar{A}_{i} \hat{x}+B_{i} u+G_{i}(y-\hat{y})\right) \\
\hat{y}=C \hat{x}
\end{array}\right.
$$

The state estimation error is given by:

$$
e=x-\hat{x}
$$

Its dynamic is:

$$
\begin{aligned}
\dot{e} & =\sum_{i=1}^{r} \mu_{i}(\hat{x})\left(\left(A_{0}-G_{i} C\right) e-G_{i} D \omega\right) \\
& +\sum_{i=1}^{r} \mu_{i}(x)\left(\Delta A_{i} x+\Delta B_{i} u\right) \\
& +\sum_{i=1}^{r}\left(\bar{A}_{i} \delta_{i}+B_{i} \Delta_{i}\right)
\end{aligned}
$$


where:

$$
\begin{aligned}
\delta_{i} & =\mu_{i}(x) x-\mu_{i}(\hat{x}) \hat{x} \\
\Delta_{i} & =\left(\mu_{i}(x)-\mu_{i}(\hat{x})\right) u
\end{aligned}
$$

The dynamic of the state estimation error describes the behavior of a perturbed system, and can be written as follows:

$$
\dot{e}=\sum_{i=1}^{r}\left(\mu_{i}(\hat{x})\left(A_{0}-G_{i} C\right) e+H_{i} \tilde{\omega}+\bar{A}_{i} \delta_{i}+B_{i} \Delta_{i}\right)
$$

where:

$$
\begin{gathered}
H_{i}=\left[\begin{array}{lll}
-\mu_{i}(\hat{x}) G_{i} D & \mu_{i}(x) \Delta A_{i} & \mu_{i}(x) \Delta B_{i}
\end{array}\right] \\
\tilde{\omega}(t)^{T}=\left[\begin{array}{lll}
\omega(t)^{T} & x(t)^{T} & u(t)^{T}
\end{array}\right]
\end{gathered}
$$

Assumption 1: In this subsection, we suppose that the following hypotheses hold:

- A1. The system (2) is assumed to be stable.

- A2. The weighting functions $\mu_{i}(x)$ are Lipschitz:

$$
\left|\mu_{i}(x)-\mu_{i}(\hat{x})\right|<\gamma_{1}|x-\hat{x}|
$$

- A3. The functions $\mu_{i}(x) x$ are Lipschitz:

$$
\left|\mu_{i}(x) x-\mu_{i}(\hat{x}) \hat{x}\right|<\gamma_{2}|x-\hat{x}|
$$

- A4. The input $u(t)$ of the system is bounded:

$$
|u(t)| \leq \beta
$$

From the assumptions (A1) and (A4), the state $x(t)$ of the system is bounded and the term $\tilde{\omega}(t)$ is bounded. Note that, in this work, observers are designed for the diagnosis of nonlinear systems, then the assumption (A1) is not restrictive.

Theorem 1. The state estimation error converges asymptotically to zero, and the $\mathscr{L}_{2}$ gain of the transfer from $\tilde{\omega}$ to $e$ is minimal if there exists positive and symmetric matrices $P$ and $Q$, gains $K_{i}$, positive scalars $\bar{\mu}, \lambda_{1}, \lambda_{2}, \varepsilon_{2}, \varepsilon_{3}, \varepsilon_{4}$ and $\sigma$ solution of the following problem:

$$
\min _{P, Q, K_{i}, \lambda_{1}, \lambda_{2}, \varepsilon_{2}, \varepsilon_{3}, \sigma, \bar{\mu}} \bar{\mu}
$$

s.t. the following conditions for all $i \in\{1, \ldots, r\}$ :

$$
A_{0}^{T} P+P A_{0}-K_{i} C-C^{T} K_{i}^{T}<-Q
$$

$$
\left[\begin{array}{cccccccccc}
M & 0 & 0 & 0 & P \bar{A}_{i} & P B_{i} & P M_{i}^{A} & P M_{i}^{B} & K_{i} D & \gamma_{1} \sigma I \\
* & M_{1 i} & 0 & 0 & 0 & 0 & 0 & 0 & 0 & 0 \\
* & * & M_{2 i} & 0 & 0 & 0 & 0 & 0 & 0 & 0 \\
* & * & * & M_{3 i} & 0 & 0 & 0 & 0 & 0 & 0 \\
* & * & * & * & -\lambda_{1} I & 0 & 0 & 0 & 0 & 0 \\
* & * & * & * & * & -\lambda_{2} I & 0 & 0 & 0 & 0 \\
* & * & * & * & * & * & -\varepsilon_{3} I & 0 & 0 & 0 \\
* & * & * & * & * & * & * & -\varepsilon_{4} I & 0 & 0 \\
* & * & * & * & * & * & * & * & -\varepsilon_{2} I & 0 \\
* & * & * & * & * & * & * & * & * & -\lambda_{2} I
\end{array}\right]
$$

where:

$$
\begin{gathered}
M=-Q+\left(\lambda_{1} \gamma_{2}^{2}+1\right) I \\
M_{1 i}=\left(-\bar{\mu}+\varepsilon_{2}\right) I
\end{gathered}
$$

$$
\begin{aligned}
& M_{2 i}=-\bar{\mu} I+\varepsilon_{3}\left(N_{i}^{A}\right)^{T} N_{i}^{A} \\
& M_{3 i}=-\bar{\mu} I+\varepsilon_{4}\left(N_{i}^{B}\right)^{T} N_{i}^{B}
\end{aligned}
$$

A star $(*)$ in a matrix indicates a transpose quantity. For example:

$\left[\begin{array}{cc}A & B \\ * & C\end{array}\right]$ stands for $\left[\begin{array}{cc}A & B \\ B^{T} & C\end{array}\right]$

The gains of the observer are derived from:

$$
G_{i}=P^{-1} K_{i}
$$

and the attenuation level is derived from:

$$
\mu=\sqrt{\bar{\mu}}
$$

Proof. To prove the convergence of the state estimation error to zero, we consider the quadratic Lyapunov function:

$$
V(e)=e^{T} P e, \quad P=P^{T}>0
$$

Its derivative with regard to time is:

$$
\dot{V}(e)=\dot{e}^{T} P e+e^{T} P \dot{e}
$$

by using (15) we obtain:

$$
\begin{aligned}
\dot{V}(e) & =\sum_{i=1}^{r}\left(\mu_{i}(\hat{x}) e^{T}\left(\Phi_{i}^{T} P+P \Phi_{i}\right) e+e^{T} P \bar{A}_{i} \delta_{i}+e^{T} P B_{i} \Delta_{i}\right. \\
& \left.+\delta_{i}^{T} \bar{A}_{i}^{T} P e+\Delta_{i}^{T} B_{i}^{T} P e+e^{T} P H_{i} \tilde{\omega}+\tilde{\omega}^{T} H_{i}^{T} P e\right)
\end{aligned}
$$

where $\Phi_{i}=\left(A_{0}-G_{i} C\right)$.

Lemma 2. For two matrices $X$ and $Y$ with appropriate dimensions, the following property holds:

$$
X^{T} Y+X Y^{T}<X^{T} \Omega^{-1} X+Y \Omega Y^{T}, \quad \Omega>0
$$

By using lemma 2 with $\Omega$ being a scalar, and the assumptions $\mathrm{A} 1, \mathrm{~A} 2$ and $\mathrm{A} 3$ we have:

$$
\begin{aligned}
e^{T} P \bar{A}_{i} \delta_{i}+\delta_{i}^{T} \bar{A}_{i}^{T} P e & <\lambda_{1} \delta_{i}^{T} \delta_{i}+\lambda_{1}^{-1} e^{T} P \bar{A}_{i} \bar{A}_{i}^{T} P e \\
& <\lambda_{1} \gamma_{2}^{2} e^{T} e+\lambda_{1}^{-1} e^{T} P \bar{A}_{i} \bar{A}_{i}^{T} P e
\end{aligned}
$$

$$
\begin{aligned}
e^{T} P B_{i} \Delta_{i}+\Delta_{i}^{T} B_{i}^{T} P e & <\lambda_{2} \Delta_{i}^{T} \Delta_{i}+\lambda_{2}^{-1} e^{T} P B_{i} B_{i}^{T} P e \\
& <\lambda_{2} \gamma_{1}^{2} \beta^{2} e^{T} e+\lambda_{2}^{-1} e^{T} P B_{i} B_{i}^{T} P e
\end{aligned}
$$

By substituting (30) and (31) in the derivative of the Lyapunov function (29), we obtain:

$$
\begin{aligned}
\dot{V}(e) & =\sum_{i=1}^{r}\left(e ^ { T } \left(\mu_{i}(\hat{x})\left(\Phi_{i}^{T} P+P \Phi_{i}\right)+\left(\lambda_{1} \gamma_{2}^{2}+\lambda_{2} \gamma_{1}^{2} \beta^{2}\right) I\right.\right. \\
& \left.+\lambda_{1}^{-1} P \bar{A}_{i} \bar{A}_{i}^{T} P+\lambda_{2}^{-1} P B_{i} B_{i}^{T} P\right) e \\
& \left.+e^{T} P H_{i} \tilde{\omega}+\tilde{\omega}^{T} H_{i}^{T} P e\right)
\end{aligned}
$$

In order to attenuate the effect of $\tilde{\omega}(t)$ on the state estimation error, we use an $\mathscr{L}_{2}$ approach. The goal is to minimize the $\mathscr{L}_{2}$ gain from $\tilde{\omega}(t)$ to the state estimation error $e(t)$ [19]:

$$
\frac{\|e\|_{2}}{\|\tilde{\omega}\|_{2}}<\mu, \quad \mu>0
$$


The state estimation error converges asymptotically to zero, and the $\mathscr{L}_{2}$ gain from $\tilde{\omega}$ to $e$ is bounded by $\mu$ if:

$$
\dot{V}(e)+e^{T} e-\mu^{2} \tilde{\omega}^{T} \tilde{\omega}<0
$$

By substituting $\dot{V}$, we obtain:

$$
\begin{aligned}
& \sum_{i=1}^{r}\left(e ^ { T } \left(\mu_{i}(\hat{x})\left(\Phi_{i}^{T} P+P \Phi_{i}\right)+\left(\lambda_{1} \gamma_{2}^{2}+\lambda_{2} \gamma_{1}^{2} \beta^{2}+1\right) I\right.\right. \\
+\quad & \left.\lambda_{1}^{-1} P \bar{A}_{i} \bar{A}_{i}^{T} P+\lambda_{2}^{-1} P B_{i} B_{i}^{T} P\right) e+e^{T} P H_{i} \tilde{\omega} \\
+ & \left.\tilde{\omega}^{T} H_{i}^{T} P e-\mu^{2} \tilde{\omega}^{T} \tilde{\omega}\right)<0
\end{aligned}
$$

The negativity of (35) is guaranteed if $\forall i \in\{1, \ldots, r\}$ :

$$
\sum_{i=1}^{r} \mu_{i}(\hat{x})\left(\Phi_{i}^{T} P+P \Phi_{i}\right)<-Q
$$

and:

$$
\begin{gathered}
e^{T}\left(-Q+\left(\lambda_{1} \gamma_{2}^{2}+\lambda_{2} \gamma_{1}^{2} \beta^{2}+1\right) I+\lambda_{1}^{-1} P \bar{A}_{i} \bar{A}_{i}^{T} P\right. \\
\left.+\quad \lambda_{2}^{-1} P B_{i} B_{i}^{T} P\right) e+e^{T} P H_{i} \tilde{\omega}+\tilde{\omega}^{T} H_{i}^{T} P e-\mu^{2} \tilde{\omega}^{T} \tilde{\omega}<0
\end{gathered}
$$

where $Q=Q^{T}>0$.

The weighting functions satisfy the convex sum property (3), thus (36) holds if the following inequalities hold:

$$
\left(A_{0}-G_{i} C\right)^{T} P+P\left(A_{0}-G_{i} C\right)<-Q, \quad \forall i \in\{1, \ldots, r\}
$$

The negativity of the quadratic form in $\left(e^{T} \quad \tilde{\omega}^{T}\right)^{T}$ in the LHS of (37) is guaranteed iff the following inequality is satisfied:

$$
\left[\begin{array}{cc}
\Psi_{i} & P H_{i} \\
H_{i}^{T} P & -\mu^{2} I
\end{array}\right]<0
$$

where:

$\Psi_{i}=-Q+\left(\lambda_{1} \gamma_{2}^{2}+\lambda_{2} \gamma_{1}^{2} \beta^{2}+1\right) I+\lambda_{1}^{-1} P \bar{A}_{i} \bar{A}_{i}^{T} P+\lambda_{2}^{-1} P B_{i} B_{i}^{T} P$ By using the definition of the matrix $H_{i}$ given in (16), the inequality (39) can be written as follows:

$$
\left[\begin{array}{cccc}
\Psi_{i} & 0 & 0 & 0 \\
0 & -\mu^{2} I & 0 & 0 \\
0 & 0 & -\mu^{2} I & 0 \\
0 & 0 & 0 & -\mu^{2} I
\end{array}\right]+W_{i}+W_{i}^{T}<0
$$

where:

$$
W_{i}=\left[\begin{array}{cccc}
0 & -\mu_{i}(\hat{x}) P G_{i} D & \mu_{i}(x) P \Delta A_{i} & \mu_{i}(x) P \Delta B_{i} \\
0 & 0 & 0 & 0 \\
0 & 0 & 0 & 0 \\
0 & 0 & 0 & 0
\end{array}\right]
$$

which can be decomposed as follows, taking into account the definition of $\Delta A_{i}$ and $\Delta B_{i}$ given in (5):

$W_{i}=\left[\begin{array}{cccc}0 & P G_{i} D & P M_{i}^{A} & P M_{i}^{B} \\ 0 & 0 & 0 & 0 \\ 0 & 0 & 0 & 0 \\ 0 & 0 & 0 & 0\end{array}\right]\left[\begin{array}{cccc}0 & 0 & 0 & 0 \\ 0 & -\mu_{i}(x) I & 0 & 0 \\ 0 & 0 & F_{A}(t) N_{i}^{A} & 0 \\ 0 & 0 & 0 & F_{B}(t) N_{i}^{B}\end{array}\right]$

where $F_{A}(t)=\mu_{i}(x) \Sigma_{A}(t)$ and $F_{B}(t)=\mu_{i}(x) \Sigma_{B}(t)$. The properties of the weighting functions and the terms $\Sigma_{A}(t)$ and $\Sigma_{B}(t)$ allow to deduce:

$$
\begin{aligned}
& \mu_{i}(\hat{x})^{T} \mu_{i}(\hat{x}) \leq 1 \\
& F_{A}(t)^{T} F_{A}(t) \leq I \\
& F_{B}(t)^{T} F_{B}(t) \leq I
\end{aligned}
$$

Let define $\Omega$ as:

$$
\begin{gathered}
\Omega=\operatorname{diag}\left(\varepsilon_{1} I, \varepsilon_{2} I, \varepsilon_{3} I, \varepsilon_{4} I\right) \\
\varepsilon_{1}>0, \varepsilon_{2}>0, \varepsilon_{3}>0, \varepsilon_{4}>0
\end{gathered}
$$

By using the lemma 2 and the definitions (5)-(6), we obtain the following inequality after some calculations:

$$
W_{i}+W_{i}^{T}<\left[\begin{array}{cccc}
\Xi_{i} & 0 & 0 & 0 \\
0 & \varepsilon_{1} I & 0 & 0 \\
0 & 0 & \varepsilon_{3}\left(N_{i}^{A}\right)^{T} N_{i}^{A} & 0 \\
0 & 0 & 0 & \varepsilon_{4}\left(N_{i}^{B}\right)^{T} N_{i}^{B}
\end{array}\right]
$$

where:

$$
\Xi_{i}=\varepsilon_{2}^{-1} P G_{i} D D^{T} G_{i}^{T} P+\varepsilon_{3}^{-1} P M_{i}^{A}\left(M_{i}^{A}\right)^{T} P+\varepsilon_{4}^{-1} P M_{i}^{B}\left(M_{i}^{B}\right)^{T} P
$$

Substituting (43) in (40) we obtain:

$$
\left[\begin{array}{cccc}
\Theta_{i} & 0 & 0 & 0 \\
0 & M_{1 i} & 0 & 0 \\
0 & 0 & M_{2 i} & 0 \\
0 & 0 & 0 & M_{3 i}
\end{array}\right]<0
$$

$$
\begin{aligned}
\Theta_{i} & =-Q+\left(\lambda_{1} \gamma_{2}^{2}+\lambda_{2} \gamma_{1}^{2} \beta^{2}+1\right) I+\lambda_{1}^{-1} P \bar{A}_{i} \bar{A}_{i}^{T} P \\
& +\lambda_{2}^{-1} P B_{i} B_{i}^{T} P+\varepsilon_{2}^{-1} P G_{i} D D^{T} G_{i}^{T} P+\varepsilon_{3}^{-1} P M_{i}^{A}\left(M_{i}^{A}\right)^{T} P \\
& +\varepsilon_{4}^{-1} P M_{i}^{B}\left(M_{i}^{B}\right)^{T} P
\end{aligned}
$$

where $M_{1 i}, M_{2 i}$ and $M_{3 i}$ are given in (22)-(24).

To eliminate the nonlinearities in (45), we use the Schur complement and the changes of variables $K_{i}=P G_{i}$. In addition, we consider that the constant $\mu$ in (44) is a variable that can be minimized in order to minimize the transfer from $\tilde{\omega}$ to the state estimation error $e$, so, we perform the change of variable $\bar{\mu}=\mu^{2}$ to eliminate the nonlinearity.

Notice that the bound of the input can also be considered as a variable and can be optimized. Let us denote $\rho$ this bound. So, we introduce another nonlinearity between $\lambda_{2}$ and $\rho$ which is removed by using the Schur complement and the change of variable $\sigma=\lambda_{2} \rho$.

The bound $\rho$ :

$$
\rho=\frac{\sigma}{\lambda_{2}}
$$

must be greater or equal to the real bound $\beta$, so we introduce the following constraint on $\sigma$ :

$$
\frac{\sigma}{\lambda_{2}}>\beta
$$

Thus we obtain the following linear constraint:

$$
\sigma-\lambda_{2} \beta>0
$$

Finally, the convergence of the state estimation error is guaranteed if the inequalities (38), (44) and (48) hold. By using the Schur complement in (44), we obtain the conditions expressed in the theorem 1 .

Numerical example and simulations. Let us consider the system in (4) defined by:

$$
A_{1}=\left[\begin{array}{ccc}
-18.5 & 5 & 18.5 \\
0 & -20.9 & 15 \\
18.5 & 15 & -33.5
\end{array}\right], A_{2}=\left[\begin{array}{ccc}
-22.1 & 0 & 22.1 \\
1 & -23.3 & 17.6 \\
17.1 & 17.6 & -39.5
\end{array}\right]
$$




$$
\begin{gathered}
B_{1}=\left[\begin{array}{c}
1 \\
0.5 \\
0.5
\end{array}\right], B_{2}=\left[\begin{array}{c}
0.5 \\
1 \\
0.25
\end{array}\right], C=\left[\begin{array}{lll}
1 & 1 & 1 \\
1 & 0 & 1
\end{array}\right], D=\left[\begin{array}{l}
0.3 \\
0.9
\end{array}\right] \\
M_{i}^{A}=N_{i}^{A}=\left[\begin{array}{lll}
0.1 & 0.1 & 0.1 \\
0.1 & 0.1 & 0.1 \\
0.1 & 0.1 & 0.1
\end{array}\right], M_{i}^{B}=N_{i}^{B}=\left[\begin{array}{l}
0.1 \\
0.1 \\
0.1
\end{array}\right], i=1,2
\end{gathered}
$$

The weighting functions are defined as follows:

$$
\left\{\begin{array}{l}
\mu_{1}(x)=\frac{1-\tanh \left(x_{1}\right)}{2} \\
\mu_{2}(x)=1-\mu_{1}(x)
\end{array}\right.
$$

$\Sigma_{A}$ and $\Sigma_{B}$ are identical and depicted in the figure 1.

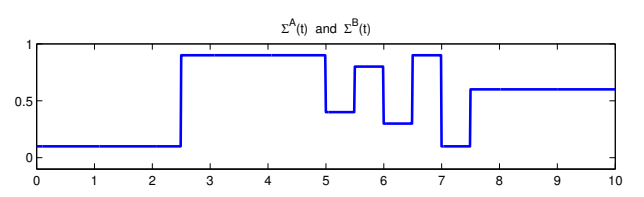

Fig. 1. $\Sigma_{A}(t)$ and $\Sigma_{B}(t)$

The LMIs in theorem 1 are satisfied with:

$$
\begin{gathered}
G_{1}=\left[\begin{array}{ll}
60.34 & -12.92 \\
59.83 & -13.18 \\
59.35 & -12.65
\end{array}\right], \mathrm{G}_{2}=\left[\begin{array}{ll}
60.38 & -12.97 \\
59.86 & -13.23 \\
59.40 & -12.71
\end{array}\right] \\
P=\left[\begin{array}{ccc}
0.76 & -0.26 & -0.41 \\
-0.26 & 0.68 & -0.32 \\
-0.41 & -0.32 & 0.83
\end{array}\right]
\end{gathered}
$$

The resulting attenuation level is $\mu=0.0083$. The simulation results are depicted in the figures 2 and 3 . We can conclude that the gains which are obtained by solving the LMIs in theorem 1 allow to attenuate the effect of the uncertainties and the noise measurement. In the figure 3 , the state estimation error obtained by using theorem 1 converges asymptotically to zero and the effect of the perturbations is attenuated (uncertainties and noise measurement). Comparing to the state estimation error obtained by a method without taking into account the uncertainties (method proposed in [20]), we can conclude that the proposed method is robust against modeling uncertainties and measurement noise. Unfortunately, the LMIs expressed in the theorem 1 depend on the Lipschitz constants of the weighting functions and the upper bound of the system input.

The problem of theorem 1 may not have a solution for large values of these constants. So this method can be applied only with systems that satisfy the assumption 1 where the Lipschitz constant and the upper bound of the input do not exceed a given threshold in order to find a solution for the problem of theorem 1 . We therefore propose another method in the next section, where the Lipschitz property of the weighting functions and the knowledge of the upper bound of the input are not necessary.

\section{B. Second approach}

The system (4) can be represented as follows:

$$
\begin{aligned}
& \dot{x}=\sum_{i=1}^{r} \mu_{i}(\hat{x})\left(\left(A_{i}+\Delta A_{i}\right) x+\left(B_{i}+\Delta B_{i}\right) u+v\right) \\
& y=C x+D \omega
\end{aligned}
$$
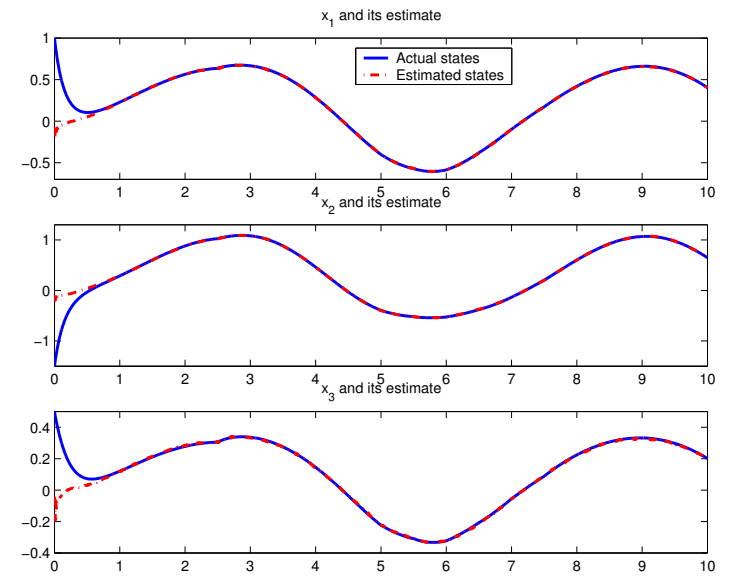

Fig. 2. State estimation
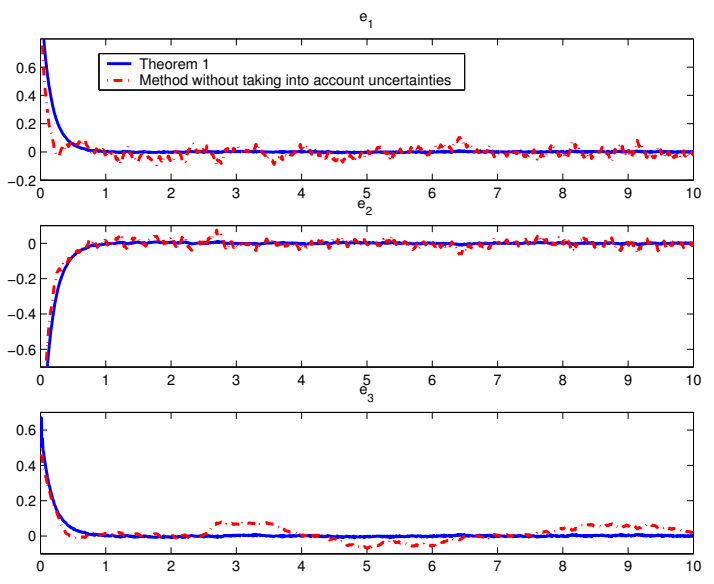

Fig. 3. State estimation error

where:

$$
v=\sum_{i=1}^{r}\left(\mu_{i}(x)-\mu_{i}(\hat{x})\right)\left(\left(A_{i}+\Delta A_{i}\right) x+\left(B_{i}+\Delta B_{i}\right) u\right)
$$

The term $v(t)$ is considered as a perturbation. The proposed observer is defined by:

$$
\begin{aligned}
& \dot{\hat{x}}=\sum_{i=1}^{r} \mu_{i}(\hat{x})\left(A_{i} \hat{x}+B_{i} u+G_{i}(y-\hat{y})\right) \\
& \hat{y}=C \hat{x}
\end{aligned}
$$

The state estimation error between (49) and (50) is defined as:

$$
e=x-\hat{x}
$$

its dynamic is given by:

$$
\dot{e}=\sum_{i=1}^{r} \mu_{i}(\hat{x})\left(\left(A_{i}-G_{i} C\right) e+\Delta A_{i} x+\Delta B_{i} u-G_{i} D \omega+v\right)
$$

which can be written using the following form:

$$
\dot{e}=\sum_{i=1}^{r} \mu_{i}(\hat{x})\left(\left(A_{i}-G_{i} C\right) e+\mathscr{M}_{i} \tilde{\omega}\right)
$$

where:

$$
\tilde{\omega}=\left[\begin{array}{llll}
v^{T} & \omega^{T} & x^{T} & u^{T}
\end{array}\right]^{T}, \quad \mathscr{M}_{i}=\left[\begin{array}{llll}
I & -G_{i} D & \Delta A_{i} & \Delta B_{i}
\end{array}\right]
$$

Assume that assumptions (A1) and (A4) are satisfied, thus $\tilde{\omega}(t)$ is bounded. 
Theorem 2. The state estimation error converges asymptotically to zero, and the $\mathscr{L}_{2}$ gain of the transfer from $\tilde{\omega}$ to $e$ is minimal if there exists a positive and symmetric matrix $P$, gains $K_{i}$, positive scalars $\bar{\gamma}, \varepsilon_{1}$ and $\varepsilon_{2}$ solution of the following problem:

$$
\min _{P, K_{i}, \varepsilon_{1}, \varepsilon_{2}, \bar{\gamma}} \bar{\gamma}
$$

s.t. to the constraints for all $i \in\{1, \ldots, r\}$

$$
\left[\begin{array}{ccccccc}
\Psi_{11 i} & P & -K_{i} D & 0 & 0 & P M_{i}^{A} & P M_{i}^{B} \\
P & -\bar{\gamma} I & 0 & 0 & 0 & 0 & 0 \\
-D^{T} K_{i}^{T} & 0 & -\bar{\gamma} I & 0 & 0 & 0 & 0 \\
0 & 0 & 0 & \Psi_{33 i} & 0 & 0 & 0 \\
0 & 0 & 0 & 0 & \Psi_{44 i} & 0 & 0 \\
\left(M_{i}^{A}\right)^{T} P & 0 & 0 & 0 & 0 & -\varepsilon_{1} I & 0 \\
\left(M_{i}^{B}\right)^{T} P & 0 & 0 & 0 & 0 & 0 & -\varepsilon_{2} I
\end{array}\right]<0
$$

where:

$$
\begin{aligned}
& \Psi_{11 i}=A_{i}^{T} P+P A_{i}-C^{T} K_{i}^{T}-K_{i} C+I \\
& \Psi_{33 i}=-\bar{\gamma} I+\varepsilon_{1}\left(N_{i}^{A}\right)^{T} N_{i}^{A} \\
& \Psi_{44 i}=-\bar{\gamma} I+\varepsilon_{2}\left(N_{i}^{B}\right)^{T} N_{i}^{B}
\end{aligned}
$$

The gains of the observer are derived from $G_{i}=P^{-1} K_{i}$, and the attenuation level is derived from $\gamma=\sqrt{\bar{\gamma}}$.

Element of proof. The goal is to minimize the effect of $\tilde{\omega}(t)$ on the state estimation error. So, by using the Bounded Real Lemma (BRL)[19], we obtain:

$$
\sum_{i=1}^{r} \mu_{i}(\hat{x}(t))\left[\begin{array}{cc}
\left(A_{i}-G_{i} C\right)^{T} P+P\left(A_{i}-G_{i} C\right)+I & P \mathscr{M}_{i} \\
\mathscr{M}_{i}^{T} P & -\gamma^{2} I
\end{array}\right]<0
$$

with the changes of variables $K_{i}=P G_{i}$ and $\bar{\gamma}=\gamma^{2}$, we obtain:

$$
\left[\begin{array}{cc}
A_{i}^{T} P+P A_{i}-C^{T} K_{i}^{T}-K_{i} C+I & P \mathscr{M}_{i} \\
\mathscr{M}_{i}^{T} P & -\bar{\gamma} I
\end{array}\right]<0, \forall i \in\{1, \ldots, r\}
$$

According to the definition of $\mathscr{M}_{i}$, the inequality (56) can be written in the form:

$$
\left[\begin{array}{ccccc}
\Psi_{11 i} & P & -P G_{i} D & P \Delta A_{i} & P \Delta B_{i} \\
P & -\bar{\gamma} I & 0 & 0 & 0 \\
-D^{T} G_{i}^{T} P & 0 & -\bar{\gamma} I & 0 & 0 \\
\Delta A_{i}^{T} P & 0 & 0 & -\bar{\gamma} I & 0 \\
\Delta B_{i}^{T} P & 0 & 0 & 0 & -\bar{\gamma} I
\end{array}\right]<0
$$

By using the same approach and calculation like (40)-(43) in the previous proof, we obtain the LMI conditions (54).

In theorem 2 , the bound of the input $\beta$ and the Lipschitz constants $\gamma_{1}$ and $\gamma_{2}$ defined in assumption 1 do not appear in LMIs (54). So, we can conclude that the second method is more general because it can be used even if the informations in the assumption 1 are not available unlike in the first method. Applying the observer proposed in the second method to the previous example lead to similar result, thus they are omitted.

\section{CONCLUSIONS AND FUTURE WORKS}

In this paper, two methods are proposed to estimate the state of nonlinear systems described by multiple models with unmeasurable decision variables. This structure allows to have an exact representation of the nonlinear behavior of the system, and in the context of diagnosis systems, only one multiple model is sufficient to develop observer banks to detect and isolate sensor and actuator faults. The two methods proposed here are developed in order to estimate the state of the system despite the presence of modeling uncertainties and measurement noise. The convergence conditions of the observer are established by using the second method of Lyapunov and an $\mathscr{L}_{2}$ approach. Future works will concern the application of these methods in order to construct robust residual generator for diagnosis of nonlinear systems.

\section{REFERENCES}

[1] D. Luenberger, "An introduction to observers," IEEE Transactions on Automatic Control, vol. 16, pp. 596-602, 1971.

[2] R. Kalman, "A new approach to linear filtering and prediction problems," Transactions of the ASME - Journal of Basic Engineering, vol. 82, pp. 35-45, 1960.

[3] S. Wang, E. Davison, and P. Dorato, "Observing the states of systems with unmeasurable disturbances," IEEE Transactions on Automatic Control, vol. 20, pp. 716-717, 1975.

[4] K. A. Barbosa, C. E. de Souza, and A. Trofino, "Robust filtering for uncertain linear systems: Lmi based methods with parametric lyapunov functions," Systems \& Control Letters, vol. 54, no. 3, pp. 251-262, Mar. 2005.

[5] Z. Duan, J. Zhang, C. Zhang, and E. Mosca, "Robust $H_{2}$ and $H_{\infty}$ filtering for uncertain linear systems," Automatica, vol. 42, no. 11, pp. 1919-1926, Nov. 2006.

[6] J. Chen, R. Patton, and H. Zhang, "Design of unknown input observers and robust fault detection filters," International Journal of Control, vol. 63 , no. 1 , pp. $85-105$, January 1996.

[7] X. Ding and P. Frank, "Fault detection via optimally robust detection filters," in 28th IEEE Conference on Decision and Control, 1989.

[8] R. Rajamani, "Observers for Lipschitz nonlinear systems," IEEE Transactions on Automatic Control, vol. 43, pp. 397-401, March 1998.

[9] A. Pertew, H. Marquez, and Q. Zhao, "LMI-based sensor fault diagnosis for nonlinear lipschitz systems," Automatica, vol. 43, pp. 1464-1469, 2007.

[10] M. Abbaszadeh and H. Marquez, "Robust $H_{\infty}$ observer design for a class of nonlinear uncertain systems via convex optimization," in American Control Conference, ACC'07, 2007.

[11] T. Takagi and M. Sugeno, "Fuzzy identification of systems and its applications to modeling and control," IEEE Transactions on Systems, Man, and Cybernetics, vol. 15, pp. 116-132, 1985.

[12] K. Tanaka, T. Ikeda, and H. Wang, "Fuzzy regulators and fuzzy observers: Relaxed stability conditions and LMI-based designs," IEEE Transactions on Fuzzy Systems, vol. 6, no. 2, pp. 250-265, 1998.

[13] A. Kruzewski, R. Wang, and T.-M. Guerra, "Non-quadratic stabilization conditions for a class of uncertain non linear discrete-time TS fuzzy models: a new approach," IEEE Transactions on Automatic Control, vol. 53, no. 2, pp. 606-611, 2008.

[14] R. Patton, J. Chen, and C. Lopez-Toribio, "Fuzzy observers for nonlinear dynamic systems fault diagnosis," in 37th IEEE Conference on Decision and Control, Tampa, Florida USA, 1998.

[15] A. Akhenak, M. Chadli, J. Ragot, and D. Maquin, "Design of sliding mode unknown input observer for uncertain Takagi-Sugeno model," in 15th Mediterranean Conference on Control and Automation, MED'07, 2007.

[16] P. Bergsten and R. Palm, "Thau-Luenberger observers for TS fuzzy systems," in 9th IEEE International Conference on Fuzzy Systems, FUZZ IEEE 2000, San Antonio, TX, USA, 2000.

[17] R. Palm and D. Driankov, "Towards a systematic analysis of fuzzy observers," in 18th NAFIPS Conference, New York, NY, USA, 1999.

[18] P. Bergsten, R. Palm, and D. Driankov, "Observers for Takagi-Sugeno fuzzy systems," IEEE Transactions on Systems, Man, and Cybernetics - Part B: Cybernetics, vol. 32, no. 1, pp. 114-121, 2002.

[19] S. Boyd, L. El Ghaoui, E. Feron, and V. Balakrishnan, Linear Matrix Inequalities in System and Control Theory, P. SIAM, Ed., 1994.

[20] D. Ichalal, B. Marx, J. Ragot, and D. Maquin, "Conception d'observateurs pour un modèle de Takagi-Sugeno à variables de décision non mesurables," in Eighth international Conference on Sciences and Techniques of Automatic control, STA'2007, Sousse, Tunisia, November 05-07 2007. 